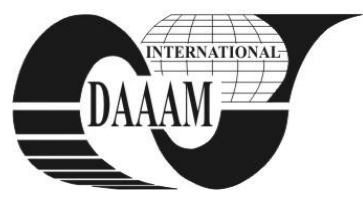

Annals of DAAAM for 2011 \& Proceedings of the 22nd International DAAAM Symposium, Volume 22, No. 1, ISSN 1726-9679 ISBN 978-3-901509-83-4, Editor B. Katalinic, Published by DAAAM International, Vienna, Austria, EU, 2011 Make Harmony between Technology and Nature, and Your Mind will Fly Free as a Bird Annals \& Proceedings of DAAAM International 2011

\title{
VIZUALIZATION OF EVOLUTIONARY ALGORITHMS BY MEANS OF COMPLEX NETWORKS
}

\author{
KLIMKOVA, E[va] \& FEKIAC, J[ozef]
}

\begin{abstract}
This paper described the relations between algorithm and complex networks. The main task of this paper is to explain how to visualize the dynamics of evolution algorithm by means of complex networks. Twenty experiments were performed for various settings and different test functions were used.

Key words: complex network, directed network, evolutionary algorithm, genetic algorithm
\end{abstract}

\section{INTRODUCTION}

In this article will be studed the relations between algorithms and complex network.

Complex network can be found in both many man-made system and nature for example network of citations of scientific papers, communication network, network of collaboration, telephone call graph, neural network, network of metabolic reaction etc. These networks are characterized by non-trivial topology and structure that is neither regular nor purely random (Dorogovtsev \& Mendes, 2003) ( Bornholdt \& Shuster, 2003) (Boccaletti, 2006). Their features include a heavy tail in degree distribution, a high clustering coefficient, hierarchical structure. It is important to understand to the topological structure of networks and its changes under external action. Then, it is possible to understand, where the network is vulnerable to damage and when are resistant.

Evolutionary algorithmus are one of the chapters of computer science whith is inspired by nature. This branch originated on beginning of the second half of the 20th century. Ideas of Evolutionary algorithm have been published (Turing, 1969) and boom occurred after the publication of Genetic Algorithms (GA) (Holland, 1975). Futher followed Evulutionary Strategy (EC) (Schwefel, 1974) (Rechenberg, 1971) and Evolutionary Programing (EP) (Fogel, 1998).

Task of this article is to demonstrate how to visualize the dynamics of Evolutionary algorithmus using Complex network. This work follows on research (Zelinka, 2011). Possibilities of its use are discussed at the end.

\section{EXPERIMENT DESIGN}

\subsection{Selected algorithms and its settings}

For experiment Genetic Algorithm was used though GA is quite old and are inferior toother newer algorithms. GA was chosen as an example of clascical evolutionary algorithm, which uses intersection of genes and mutations for the next generation.

The experiment was repeated twenty times for each settings of the GA to confirm the robustness and efficiency of developed algorithm. For the experiment, desktop PC with single-core, $1.81 \mathrm{GHz}$ CPU and $2 \mathrm{~GB}$ RAM was used. For all calculations and data processing, Mathematica version 7.0.0 was used.

The asynchronous mode of GA corresponds to picking the parents, do crossover and mutation Algorithm compares the children with the parents and selects the best of them. Settings
AG are given in Tab. 1, they are chosen such that algorithm find extrema in an acceptable time.

\begin{tabular}{|c|c|}
\hline String length & 20 \\
\hline Mutation rate & 0.2 \\
\hline Pop size & $5-50$ \\
\hline Generations & $50-300$ \\
\hline
\end{tabular}

Tab. 1. Parametr seting of Genatic Algorithm

\subsection{Tests function}

For the purposes of visualization of complex network were selected following four functions. $1^{\text {st }}$ De Jong's function (1), 3 rd De Jong' s function (2), Stretched V sine wave function (Ackley) (3), and Schwefel's function (4). All function are dispalid in figure 1 . These functions are very often used by scientists working with evolutionary algorithms.

$$
\begin{gathered}
f(x)=\sum_{i=1}^{n} x_{i}^{2} \\
f(x)=\sum_{i=1}^{n}\left|x_{i}\right| \\
f(x)=\sum_{i=1}^{n}\left(x_{i+1}^{2}+x_{i}^{2}\right)^{0.25}\left(\sin ^{2}\left(50\left(x_{i+1}^{2}+x_{i}^{2}\right)^{0.1}\right)+1\right)
\end{gathered}
$$

$$
f(x)=\sum_{i=1}^{n}\left[-x_{i} \sin \left(\sqrt{\left|x_{i}\right|}\right)\right]
$$

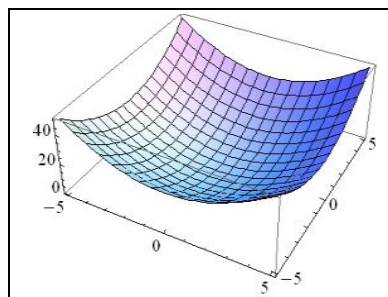

(a)

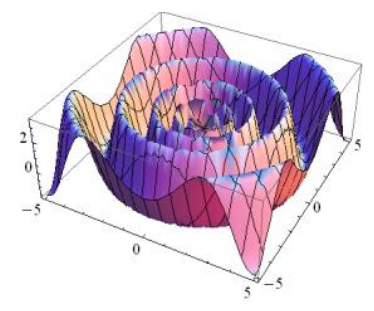

(c)

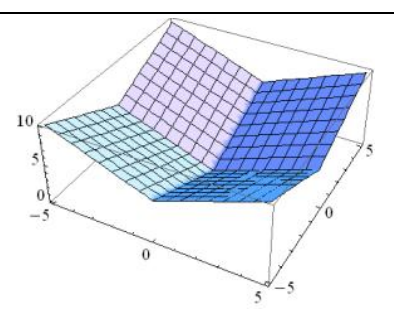

(b)

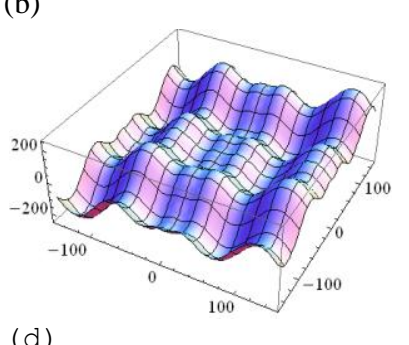

Fig. 1. Test function (a) $1^{\text {st }}$ De Jong' s function, (b) $3 \mathrm{rd}$ De Jong' s function, (c) Ackley, (d) Schwefel's function

\subsection{Vizualization complex network}

One of the most important thing is how the selected data to display in complex network. The main vision was such that each individual is represented by vertex and edges between 
vertices should reflect dynamics in population, i.e. interactions between individuals (which individual has been succesfully used for offspring creation). In genetic algorithms there are generated 2 new individuals crossing of two parents. Than parents with children compete for space in the new generation. Each individual that is added to the next population, is represented by new vertex that is addend to the network. Relations between parents and offspring are reprezentated by edges.

\section{EXPERIMENT RESULTS}

Twenty experiments were performed for different settings of GA: popSize and Generations. Genetic Algorithm was tested on four test function with constant dimensions. Experimental results were processed graphically and statistically. Emergences of complex network structure behind evolutionary dynamics depend on many factors, though some special versions of used algorithms did not show complex network structure, despite the fact that the number of generations was quite large.

Figure 2 shows the interaction between individuals, that create complex network structure, that heaving properties of Small World. The proof of these properties is long tail distribution of vertex that is shown in Fig. 3.

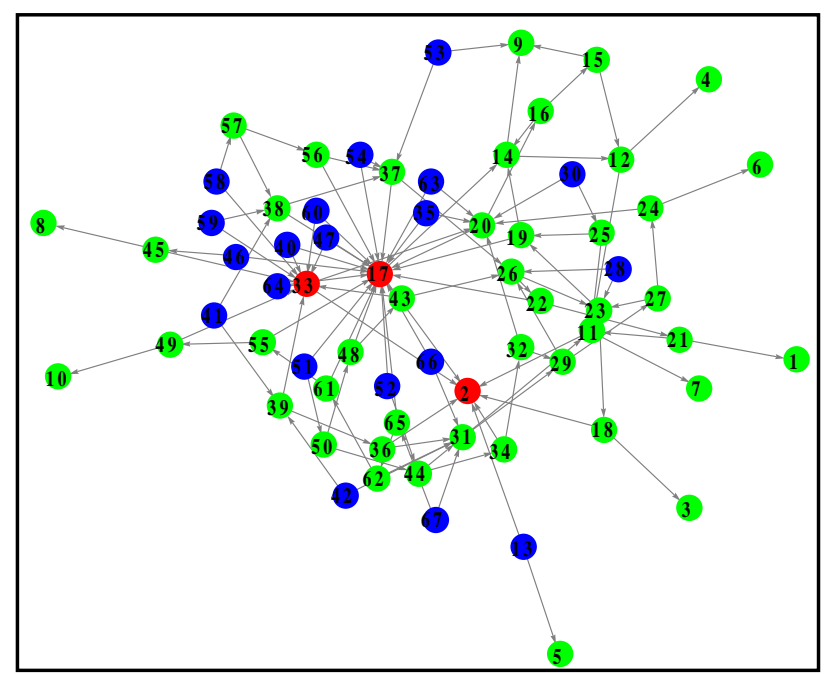

Fig. 2. Complex network structure demonstrates relatipnship between individuals. Red vertexes have the most input edges and blue vertexes don't have input edges

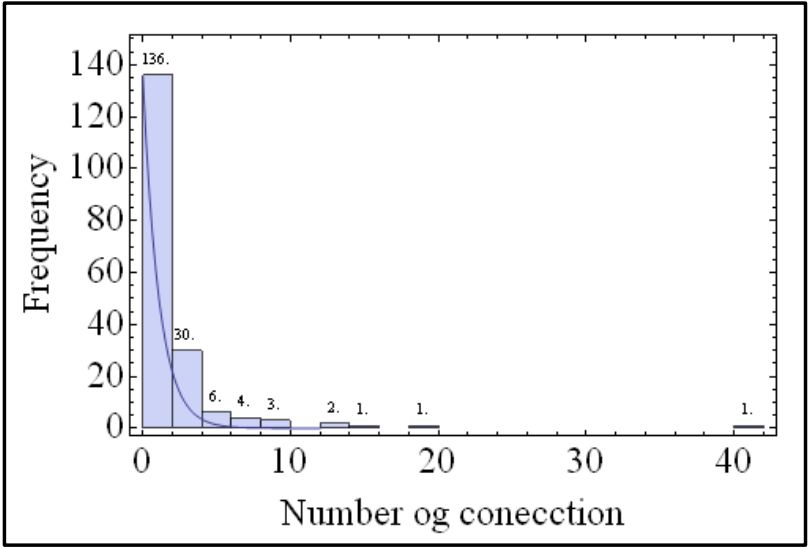

Fig. 3. An example of histogram exhibiting long tail distribution of vertices degree

The increasing of complex network structure (CNS) is dependent on the number of generations. With a low number of generations does not rise to the CNS.
The dependence between the test function and the increase of the CNS has not exactly found. However when more complex function were using, as Ackley's function, it needed more generations or large population to obtain CNS. Whereas eased fiction was using as De Jong' s function, CNS was observed already at a low number of generations. Progress of Genetic algorithm is shown in Fig. 4.

As alredy mentioned, the size of population or Pop size has affects the formation CNS. At very small population formation of CNS was not observed. Again, it is the parameter, which does not influent CNS forming alone, but in a combination with another parameters, as mentioned in the previous items.

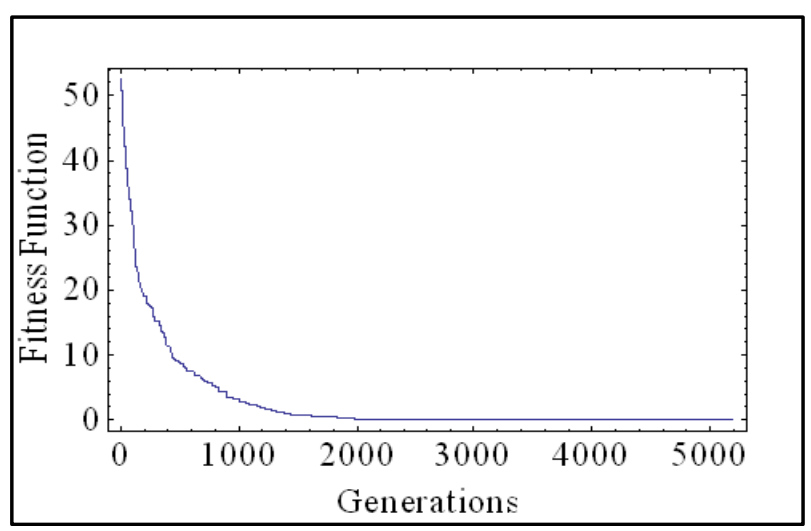

Fig. 4. An example of activated Leaders at the moment when evolution has found the global extreme

\section{CONCLUSION}

The results reported here are based on (Zelinka, 2011) and should be treated as preliminary. It will require more robust simulations and more statistical calculations to obtain further conclusions.

\section{ACKNOWLEDGEMENTS}

This work was supported by the grants: Internal Grant Agency of Tomas Bata University under the project No. IGA/40/FAI/11/D.

\section{REFERENCES}

Boccaletti. S.: et al. (2006). Complex Networks: Structure and Dynamics, Phys. Rep. 424, 175-308

Bornholdt, S.: Shuster, H. G. (2003). Handbook of Graphs and Networks, WILEY-VCH

Dorogovtsev, S.N.: Mendes, J.F.F. (2003). Evolution of Networks, Oxford University press

Fogel,D.B.: (1998). Unearthinga Fossil from the History of Evolutionary Computation. Fundamenta Informaticae 35(14), 1-16

Holland, J.: (1975). Adaptation in natural and artificial systems. Univ. of Michigan Press, Ann Arbor

Rechenberg, I.: (1971) Evolutionsstrategie - Optimierung technischer Systeme nach Prinzipien der biologischen Evolution (PhD thesis), Printed in Fromman-Holzboog (1973)

Schwefel, H.: (1977) . Numerische Optimierung von ComputerModellen, Birkhauser

Turing, A. M.: (1952) The Chemical Basis of Morphogenesis, Philosophical Transactions of the Royal Society of London. Series B, Biological Sciences, Vol. 237, No. 641. (Aug. 14, 1952), pp. 37-72

Zelinka I.: Davendra D. (2011) Investigation on Relations Between Complex Networks and Evolutionary Algorithm Dynamics, IJCISIM 